\title{
High-Cycle Fatigue Properties and Damage Mechanism of Q345B Structural Steel
}

\author{
X. L. Hu, ${ }^{a}$ Y. J. Liu, ${ }^{\text {,11 }}$ M. K. Khan, ${ }^{b}$ and Q. Y. Wang ${ }^{a, c, 2}$ \\ a Key Laboratory of Energy Engineering Safety and Disaster Mechanics, Ministry of Education, \\ College of Architecture \& Environment, Sichuan University, Chengdu, China \\ ${ }^{b}$ Faculty of Engineerint and Computing, Coventry University, Coventry, UK \\ c School of Urban and Rural Construction, Chengdu University, Chengdu, China \\ ${ }^{1}$ liuyongjie@scu.edu.cn \\ 2 wangqy@scu.edu.cn
}

The high-cycle fatigue behavior of Q345B structural steel was investigated experimentally. Highfrequency vibration fatigue testing machine and scanning electron microscopy were used to study the high-cycle fatigue $S-N$ curve characteristics and crack initiation mechanism at ambient temperature. The surface temperature of the specimens was monitored. The relation between the fatigue limit and the amount of heat dissipation was also investigated. It was found that the fatigue life changed inversely with the stress amplitude in the high-cycle range. The fatigue limit in high cycle range was obtained from heat dissipation in the specimen and found to have good agreement with the $S-N$ curve. The crack initiation was attributed to the surface defects and the persistent slip bands due to the cycle slip in fatigue loading.

Keywords: Q345B structural steel, high-cycle fatigue, heat dissipation, fatigue strength, fatigue crack initiation mechanism.

Introduction. Q345B is a low-alloy steel of Chinese brand, which exhibits excellent performance in low-temperature, cold stamping, cutting, welding applications. It is widely used in bridge construction, automotive components, pressure vessels etc. In bridge civil engineering bridge applications, the material experiences static and dynamic loading due to vehicles and wind. The dynamic loads are of low level but act for large amount of cycles. If the structural components in this application are not designed appropriately, the cumulative damage due to all these loadings result in the fatigue fracture, which may be of serious consequences.

In structural components, the commonly used high strength steel $[1,2]$, low carbon steel $[3,4]$ and stainless steel $[5,6]$ alloys, the eariler studies mainly focused on low-cycle fatigue where the fatigue cycles mainly remain less than $10^{5}$ cycles. Recent studies have shown that for many engineering alloys the concept of traditional fatigue limit is not very accurate. The fatigue failure occur after high-cycle periodic loads beyond $10^{7}$ cycles. Under these conditions, the stresses are found lower than the yield strength. Other parameters have been studied to investigate the high-cycle fatigue behavior of structural steels [7-12] such as $S-N$ curves, fatigue strength, fatigue life prediction, structure evolution law, fatigue crack initiation and propagation mechanism. The effect of microstructure, prestrain, average stress, material defects (inclusions), gaps and surface treatments, on the cracking behaviour of the material have also been studied. However, the research on the high cycle fatigue behavior of Q345B structural steel is still scarce at present. This study used high-frequency tension and compression load to study the high-cycle fatigue performance of Q345B structural steel beyond $10^{5}$ cycles. The fatigue fracture of specimens was observed by electron microscope and the fatigue cracks initiation mechanism was investigated. The surface temperature of the specimens during the high-frequency tension and compression 
progress was monitored and analyzed by infrared imager. The relationship between the heat dissipation amount and fatigue limits was discussed to acquire the fatigue limit of the material.

1. Test Materials and Specimens. The commercial hot-rolled Q345B low alloy steel of thickness of $20 \mathrm{~mm}$ was used in this study. The main chemical compositions and mechanical properties of the material are shown in Tables 1 and 2, respectively. The microstructure of the material is shown in Fig. 1. The material is a rolled ferrite pearlite dual phase low carbon steel, and the two phases are in zonal distribution. The microstructure content of lamellar hard pearlite is about $30 \%$, and the ferrite content is about $70 \%$. Due to the low carbon content in the material composition, the ferrite phase was abundant while pearlite was scarce in the microstructure. The strength and hardness of ferrite was not high however, the material showed good ductility and toughness.

$\mathrm{T}$ a b 1 e 1

Chemical Composition of Q345B Steel (wt.\%)

\begin{tabular}{|c|c|c|c|c|}
\hline $\mathrm{C}$ & $\mathrm{Si}$ & $\mathrm{Mn}$ & $\mathrm{S}$ & $\mathrm{P}$ \\
\hline 0.16 & 0.35 & 1.34 & 0.11 & 0.22 \\
\hline
\end{tabular}

$\mathrm{T}$ a b 1 e 2

Mechanical Properties of Q345B Steel

\begin{tabular}{|c|c|c|c|c|}
\hline$E, \mathrm{GPa}$ & $\sigma_{s}, \mathrm{MPa}$ & $\sigma_{b}, \mathrm{MPa}$ & $\rho, \mathrm{g} / \mathrm{cm}^{3}$ & $A$ \\
\hline 201 & 395 & 550 & 7.85 & 0.27 \\
\hline
\end{tabular}

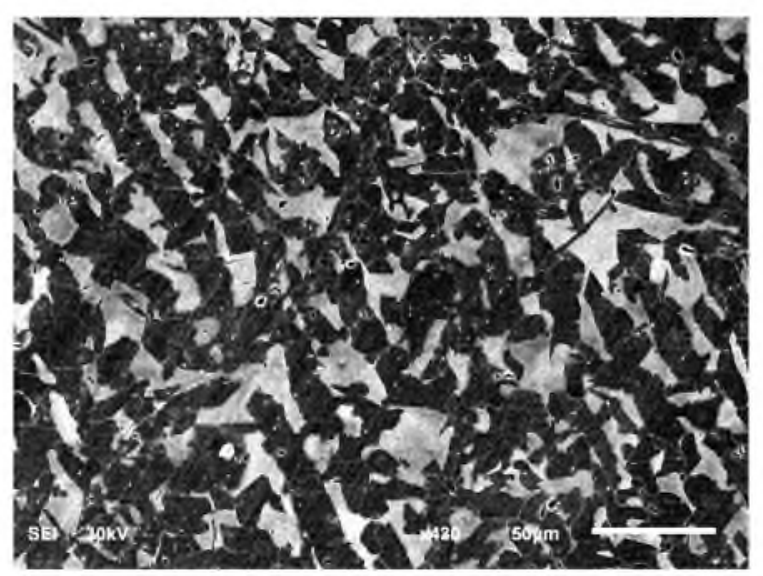

Fig. 1. Microstructure of Q345B steel.

The fatigue specimen was designed based on the resonance principle. Its size is shown in Fig. 2. The natural vibration frequency is $155 \mathrm{~Hz}$.

2. Methods.

2.1. Test Methods. The fatigue tests were carried out on the high-frequency electromagnetic vibration fatigue test machine (Changchun Qian Bang QBG-100). The test machine utilized the electromagnetic resonance principle. The loading stress ratio $R$ was set as -1 . The test frequency was determined by the aforementioned frequency of specimen and set as $155 \mathrm{~Hz}$. Cyclic loaded stresses were set from 250 to $300 \mathrm{MPa}$ at 6 levels. 


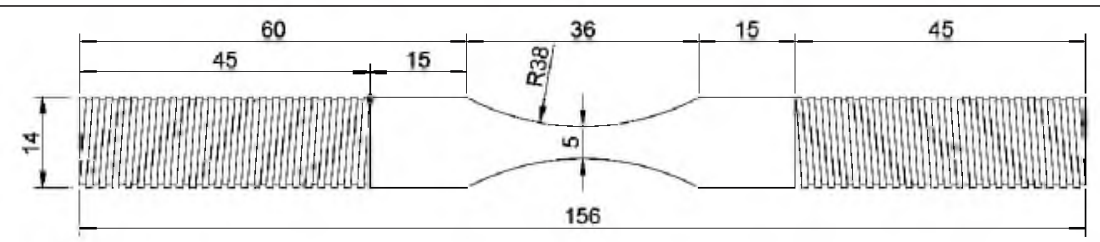

Fig. 2. Schematic diagram of the specimen dimensions (in $\mathrm{mm}$ ).

2.2. Heat Dissipation Test. As a non-contact, real-time, nondestructive measurement technology, infrared imaging can precisely measure, display and analyze the temperature distribution on the surface of the specimen. As a result, it can be applied to the heat dissipation analysis. The heat dissipation in the fatigue progress was analyzed through the temperature changes on the surface of the specimen. The measurement system is shown in Fig. 3. The temperature distribution of the specimen was monitored by an infrared imager (NEC NS9500) with an accuracy of $\pm 0.1^{\circ}$. The infrared imager was calibrated by thermocouples and mercury thermometers before the test. To avoid the errors caused by the fluctuation of ambient temperature, a test piece was placed next to the sample as a reference. So the change of the surface temperature of the test piece can be expressed as

$$
\Delta T_{a}=\Delta T_{t}-\Delta T_{r}
$$

where $\Delta T_{t}$ and $\Delta T_{r}$ are the changes of the surface temperature of the test and referenced pieces, respectively.

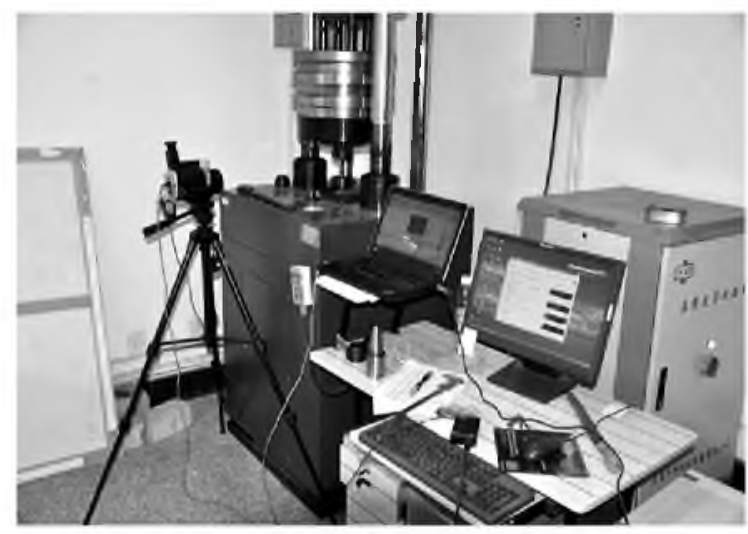

Fig. 3. Measurement of thermal dissipation.

2.3. Microscopic Analysis of Fatigue Fracture. Scanning electron microscopy (SEM) was used for microscopic observation of the fatigue fracture of the specimens. The mechanism of initiation and propagation of fatigue cracks was investigated.

2.4. Statistics of Fatigue Data. The $S-N$ curve is usually described by exponential function, power function, Basquin equation [13, 14] or three-parameter model [15]. The Basquin equation was used in this study and it is expressed as

$$
\sigma_{a}=\sigma_{f}^{\prime}\left(2 N_{f}\right)^{b}
$$

where $\sigma_{a}$ is the load amplitude, MPa, $N_{f}$ is the fatigue life, $\sigma_{f}^{\prime}$ is the coefficient of fatigue strength, $\mathrm{MPa}$, and $b$ is the exponent of fatigue strength, namely the Basquin exponent. The constants $\sigma_{f}^{\prime}$ and $b$ are obtained by the least squares method. 


\section{Results and Discussion.}

3.1. $S-N$ Curve. The tested $S-N$ data of high-frequency tension and compression fatigue for Q345B in the high-cycle range are shown in Fig. 4. As it can be seen from the figure, the fatigue life varied inversely with stress amplitude. The specimen below $240 \mathrm{MPa}$ stress did not show fracture. So the fatigue limit of Q345B in the high-cycle range was concluded as $240 \mathrm{MPa}$.

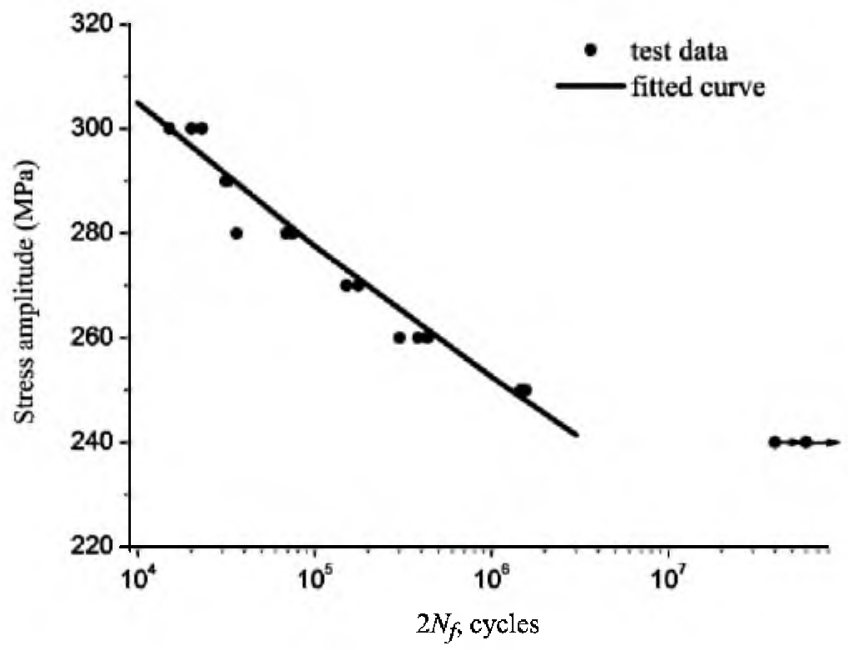

Fig. 4. $S-N$ curve for high-frequency tension and compression fatigue with high cycles of Q345 steel.

The tested $S-N$ data are fitted by Basquin equation and the result is

$$
\sigma_{a}=444.95\left(2 N_{f}\right)^{-0.041}, \quad R^{2}=0.962 .
$$

The fitted curve was plotted in Fig. 4 as solid line. It can be seen that the test data distribute around the Basquin equation, with the fitted $R$ close to 1 . So Basquin equation can quantitatively describe the features of symmetrical tension and compression fatigue $S-N$ curve in the high-cycle range for Q345B.

3.2. The Mechanism of Fatigue Crack Initiation. Fatigue fracture was observed microscopically by scanning electron microscopy. The cross-sections of fatigue specimens were all flat fractures normal to the axial stress. All the fatigue cracks initiate on the surface of specimen. More crack initiations occur under the conditions of high stress and short life.

The fracture morphologies are shown in Fig. 5 for $\sigma$ equal to $260 \mathrm{MPa}$ and $N_{f}$ equal to $7.55 \cdot 10^{4}$. The fatigue initiation zone, crack propagation zone and fracture zone can be clearly seen from the full view of fracture morphologies in Fig. 5a. The fracture surface showed a feature of radial river pattern in the crack propagation zone. The radial center was located at the fatigue crack initiation position on the surface of the specimen, which can be seen from the crack initiation zone in Fig. 5b. The fatigue crack initiation position was observed under high magnification, as shown in Fig. 5c. Strips on the surface of specimen can be seen clearly. For low-cycle and high-cycle fatigue fractures, large alternating loads result in the plastic deformation on the surface of specimen, especially in the areas with defects like machining marks, scraping scratches, inclusions or geometric discontinuity. Under the alternating loads, the material "squeezes" and "extrudes." As a consequence, slip lines or slip bands form on the surface. With the further effect of alternating loads, these slip lines or slip bands gradually extended to the interior of the material and formed persistent slip bands. Alternating slip results in the formation of slip steps, penetrated ditch 
and extruded strips on the surface of material, leading to the uneven surface and microcrack initiation. When the microcracks reached to a certain size, the fatigue cracks started to propagate and left traces in the crack propagation zone, namely fatigue striations as shown in Fig. 5d. Each step of fatigue cracks represents a forward extended "pace" under cyclic load. The crack propagation can be quantitatively analyzed according to the width of fatigue striations [16].

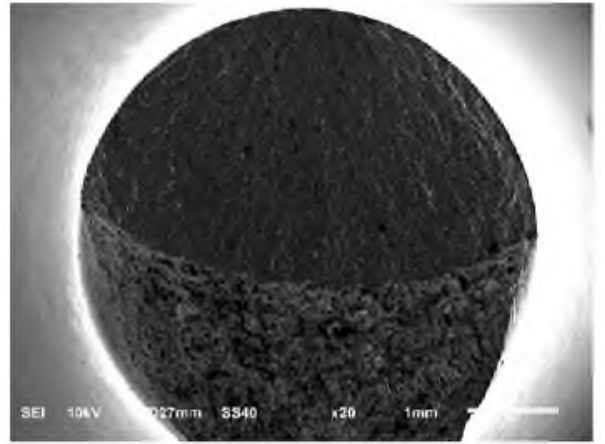

a

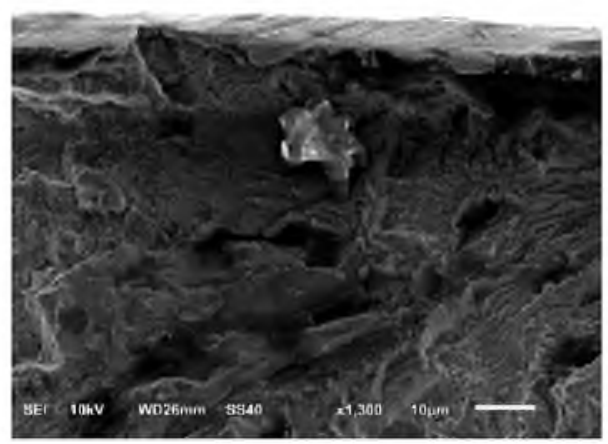

C

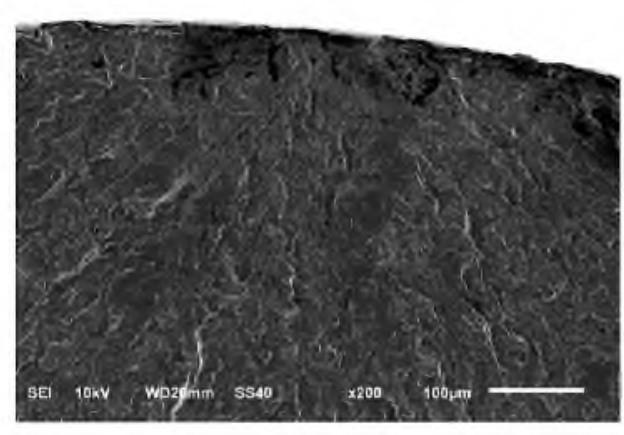

b

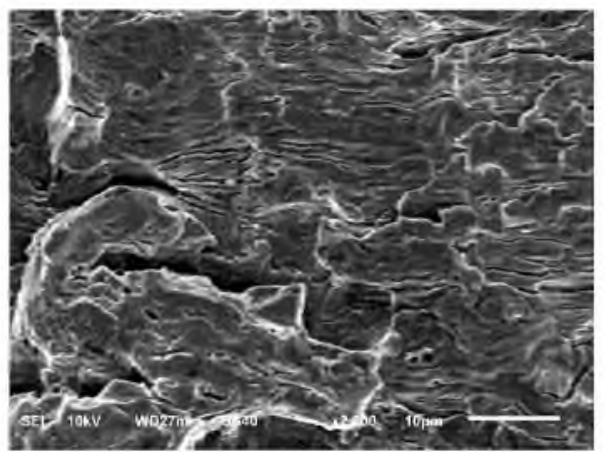

d

Fig. 5. Fatigue fracture for $\sigma=260 \mathrm{MPa}$ and $N_{f}=7.55 \cdot 10^{4}$ cycles: (a) full view of fracture morphologies; (b) fatigue initiation zone; (c) high magnification of the fatigue initiation position; (d) fatigue striations.

The fatigue cracks usually initiate at multiple positions instead of a single one on the material surface. The propagation of cracks under alternating loads leads to more crack initiation and propagation, especially under high stress. Figure 6 shows that two fatigue cracks initiate and propagate on the surface with $\sigma=280 \mathrm{MPa}$. As shown in Fig. 6b, all the fatigue cracks initiate on the material surface. The cracks expand into the interior of the material under cyclic loads. The two cracks intersect with each other at the position of the white stair. Then the stress concentration is strengthened and a large factor of stress intensity is produced at the tip of the cracks. The two cracks merge to each other to form a main crack and further accelerate the propagation.

3.3. Analysis of Heat Dissipation to Determine the Fatigue Limit. The temperature raising curves of Q345B steel in the fatigue progress are shown in Fig. 7. The specimen is under five different loads between 260 and $300 \mathrm{MPa}$. It can be seen that the fatigue progress has three typical stages, namely the initial stage of temperature rise, temperature stabilization stage and rapid temperature rising stage. The temperature changes measured by infrared imager before and after the specimens fracture are shown in Fig. 8 . The 


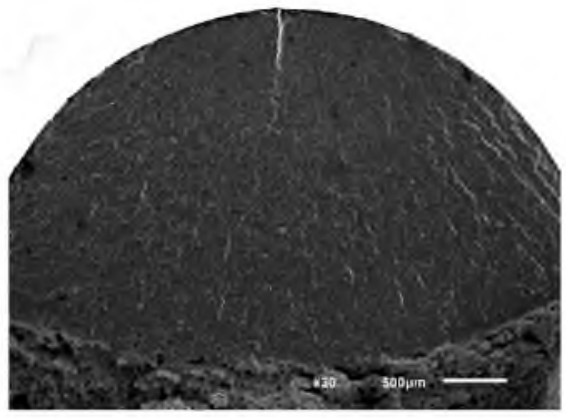

a

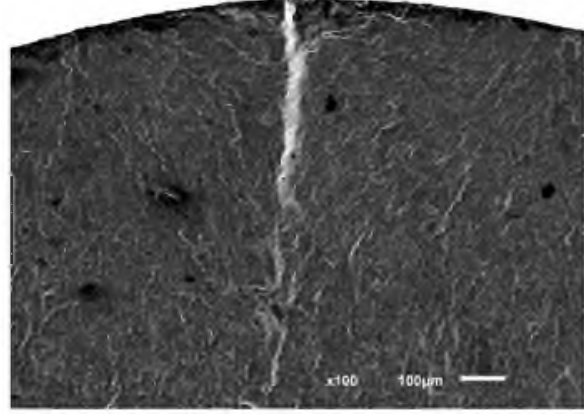

b

Fig. 6. Fatigue fracture for $\sigma=280 \mathrm{MPa}$ and $N_{f}=3.45 \cdot 10^{4}$ cycles: (a) fracture morphologies: (b) fatigue initiation zone.

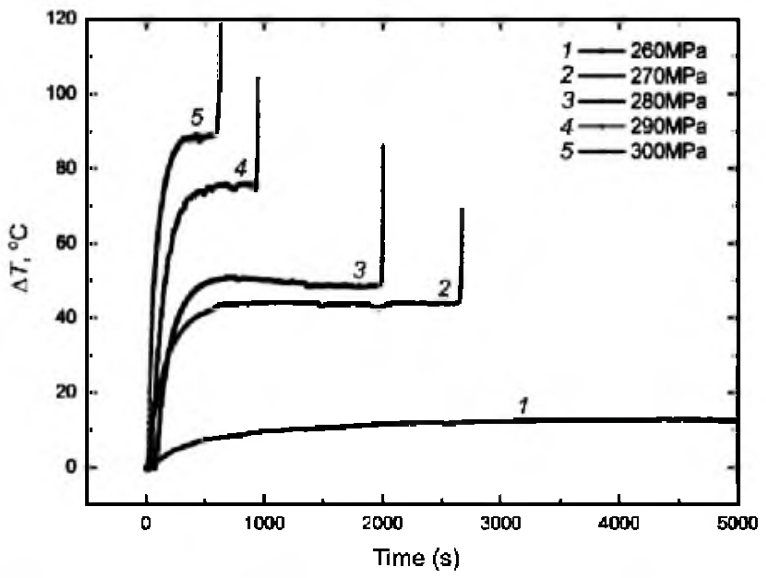

Fig. 7. Temperature rising curves of Q345B steel rate and the amplitude of load.

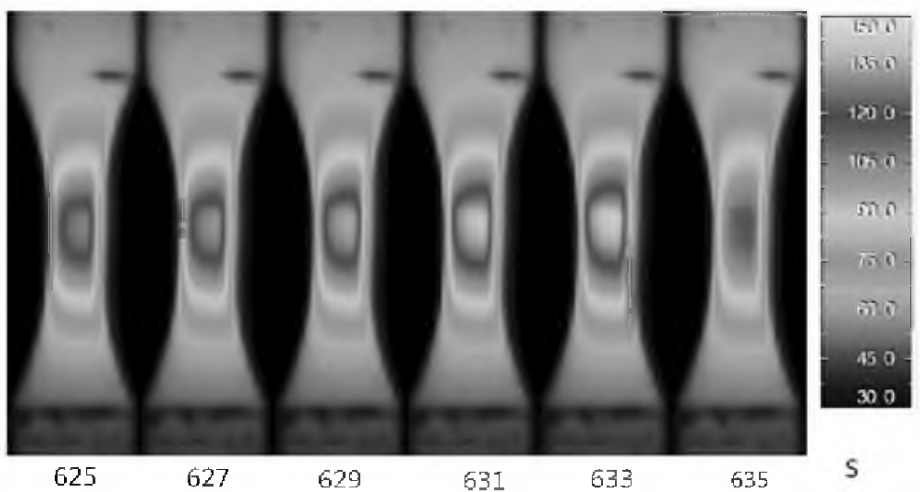

Fig. 8. Temperature change before and after the specimen fractures.

temperature rises rapidly after the cracks propagate, leading to higher temperature in the propagating zone than that in other areas. This progress corresponds to the inflection point of phase II and phase III in the temperature raising curves. The specimen fractures soon after the cracks begin to propagate. Therefore, the fatigue crack initiation is verified to be the main part of the fatigue life for high-cycle fatigue. 
Figure 8 shows that the initial and rapid temperature-rising stages take up a small part of the fatigue progress. As the amplitude of the load increases, the temperature curve at the initial temperature rising stage turned steeper. The rate of initial temperature rising increased linearly with the amplitude of load, as shown in Fig. 9. In addition, the difference between the stable temperature and initial temperature increased with the amplitude of load. The relations are plotted in Fig. 10, which shows that the temperature changes also increased linearly with the load amplitude.

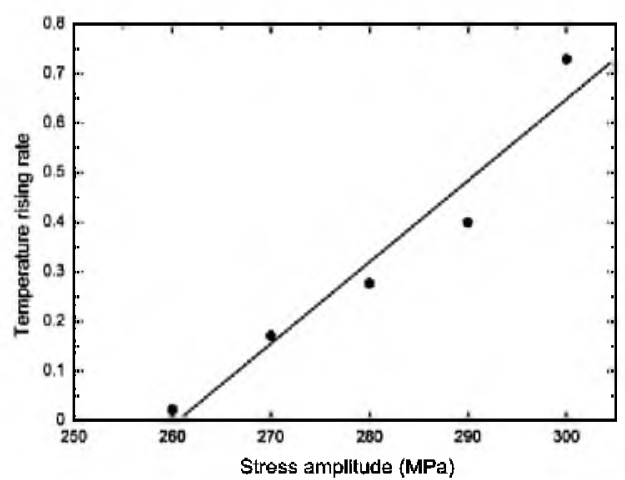

Fig. 9

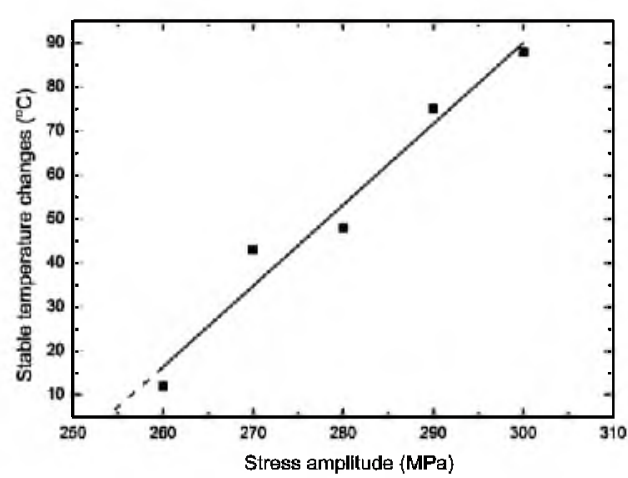

Fig. 10

Fig. 9. Relation between the temperature rising rate and the amplitude of load.

Fig. 10. Relation between the stable temperature changes and the amplitude of stress.

When the amplitude of load was smaller than the fatigue limit, the temperature change was not very evident. Therefore, the relationship between the stable temperature change and the load amplitude was obtained by linear regression according to the curve in Fig. 10. The amplitude at 0 temperature change is acquired as the fatigue limit. The relationship between the stable temperature change and the load amplitude for Q345B was linear. The intersection of the line and the $x$-axis was the fatigue limit. For Q345B steel the fatigue limit was obtained as $253 \mathrm{MPa}$, which was similar to the high-cycle fatigue limit of $240 \mathrm{MPa}$ as obtained by the $S-N$ curve.

Conclusions. The present work investigated the fatigue behavior of Q345B steel in high-cycle range by the high frequency vibration fatigue in tension and compression, measurements of infrared heat dissipation and microscopic observation. The conclusions are given below.

1. The fatigue life varied inversely with the loading stress in the range of $10^{4} \sim 10^{7}$ cycles. The material under $10^{7}$ cycles showed no fracture below $240 \mathrm{MPa}$. The fatigue limit of Q345B in the high-cycle range was obtained as $240 \mathrm{MPa}$. The test data was analyzed with Basquin equation and $S-N$ curve characteristics of structural steel Q345B was acquired.

2. The fatigue cracks under high cycle initially occurred on the surface of specimens. The stress concentration on the surface, caused by the surface defects and persistent slip bands, resulted in the cycle slip leading to the crack initiation.

3. The initial temperature rise and the stable temperature change increased linearly with the stress amplitude. A similar fatigue limit was obtained by heat dissipation analysis and $S-N$ curve.

Acknowledgments. The authors gratefully acknowledged the financial supports from the National Science Foundation of China (NSFC-11172188, 11302142) and the Program for Changjiang Scholars and Innovative Research Team (IRT14R37). 
1. Y. R. Luo, C. X. Huang, R. H. Tian, and Q.Y. Wang, "Effects of strain rate on low cycle fatigue behaviors of high-strength structural steel," J. Iron Steel Res. Int., 20, No. 7, 50-56 (2013).

2. Y. R. Luo, C. X. Huang, Y. Guo, and Q. Y. Wang, "Energy-based prediction of low cycle fatigue life of high-strength structural steel," J. Iron Steel Res. Int., 19, No. 10, 47-53 (2012).

3. X. Yan, "Low cycle fatigue and cyclic stress ratcheting failure behavior of carbon steel 45 under uniaxial cyclic loading," Int. J. Fatigue, 27, No. 9, 1124-1132 (2005).

4. L. Zong, G. Shi, and Y. Q. Wang, "Experimental investigation on fatigue crack behavior of bridge steel Q345qD base metal and butt weld," Mater. Design, 66, 196-208 (2015).

5. C. J. Miao, J. Y. Zheng, X. Z. Gao, et al., "Investigation of low-cycle fatigue behavior of austenitic stainless steel for cold-stretched pressure vessels," J. Zhejiang Univ.-Sc. $A, \mathbf{1 4}$, No. 1, 31-37 (2013).

6. K. H. Nip, L. Gardner, C. M. Davies, and A. Y. Elghazouli, "Extremely low cycle fatigue tests on structural carbon steel and stainless steel," J. Constr. Steel Res., 66, No. 1, 96-110, (2010).

7. S. Song, K. Sugimoto, S. Kandaka, et al., "Effect of prestraining on high cycle fatigue strength of high-strength low alloy TRIP-aided steels," Mater. Sci. Res. Int., 9, No. 3, 223-229 (2003).

8. K. Sugimoto, D. Fiji, and N. Yoshikawa, "Fatigue strength of newly developed high-strength low alloy TRIP-aided steels with good hardenability," Proc. Eng., 2 , No. 1, 359-362 (2010).

9. L. Vincent, J.-C. Le Roux, and S. Tahen, "On the high cycle fatigue behavior of a type 304L stainless steel at room temperature," Int. J. Fatigue, 38, 84-91 (2012).

10. N. Miura and Y. Takahashi, "High-cycle fatigue behavior of type 316 stainless steel at $288^{\circ} \mathrm{C}$ including mean stress effect," Int. J. Fatigue, 28, No. 11, 1618-1625 (2006).

11. M. Cova, P. Livieri, L. Susmel, and R. Tovo, "Defects vs small notches competition in fatigue failure initiation of cast steel," Key Eng. Mat., 417-418, 529-532 (2010).

12. N. J. Fu, J. L. Xie, C. W. Huang, and Y. Teng, "Research on fatigue properties of grade b steel casted by the resin sand process," Appl. Mech. Mater., 189, 218-224 (2012).

13. O. H. Basquin, "The exponential law of endurance tests," Proc. Annual Meeting ASTM, 10, 625-630 (1919).

14. C. C. Engler-Pinto, Jr., J. V. Lasecki, R. J. Frish, et al., "Statical approaches applied to fatigue test data analysis," Reliab. Eng. Syst. Safe, 53, No. 3, 85-90 (2005).

15. W. Weibull, Fatigue Testing and Analysis of Results, The Macmillan Company, New York (1961).

16. N. A. Fleck and R. A. Smith, "Fatigue life prediction of a structural steel under service loading," Int. J. Fatigue, 6, No. 4, 203-210 (1984). 